\title{
Retentissement sexuel, conjugal et social d'une population d'hommes stériles consultants en Andrologie : le point de vue du psychiatre de liaison
}

\author{
Michel BELLONE 1, Jean-Marc RIGOT. 2, Olivier COTTENCIN 1, Michel GOUDEMAND1 \\ 1 Clinique Fontan, CHRU de Lille. 2Service d'Andrologie, CHRU de Lille.
}

\section{RESUME}

L'infertilité est désormais abordée comme une maladie incontestablement reliée à la sexualité entraînant I'homme et son couple dans une spirale de souffrance. Aborder les interactions entre la sexualité et l'Assistance Médicale à la Procréation présenterait donc un intérêt tout particulier pour suivre et aider nos patients. Nombre d'études de la littérature avancent le fait que l'infertilité occasionne un retentissement psychologique, social et sexuel majeur pour le couple. Dans la majorité des cas ces études sont effectuées dans les services de gynécologie et sont plutôt centrées sur les retentissements féminins et conjugaux de cette infertilité.

Matériel et méthodes : L'échantillon étudié comportait 39 sujets divisés en deux groupes : 21 azoospermes et 18 oligoasthenospermes. Ces sujets ont été recrutés en consultation d'andrologie pendant une période de 6 mois. Nos sujets ont répondu à un questionnaire général sur l'infertilité décrivant le contexte clinique et symptomatique qui entoure l'infertilité, puis à un autoquestionnaire explorant le retentissement psychosocial, conjugal et sexuel de l'infertilité masculine ainsi que la représentation du psychiatre au sein d'un service d'andrologie.

Résultats : Un tiers de nos patients se sont vus annoncer pour la première fois leur diagnostic lors de la remise de leur spermogramme par le biologiste. Initialement, nos patients ont surtout envisagé des problèmes conjugaux, psychologiques et sociaux, mais très peu affectant leur sexualité. Ils se représentent l'infertilité masculine comme plus perturbante pour la femme que pour l'homme. $82 \%$ de nos sujets se représentaient leur partenaire sans difficulté sexuelle et $48,7 \%$ sans difficulté psychologique en rapport avec leur diagnostic d'infertilité. Les oligoasthénospermes se représentaient significativement plus « de difficultés psychologiques " chez leur partenaire que le groupe azoosperme. $41 \%$ de nos sujets éprouvaient un sentiment de culpabilité envers leur partenaire. Nous avions $10,3 \%$ de troubles sexuels avant le diagnostic d'infertilité masculine contre $25,6 \%$ après. II n'y a pas eu de modification importante de la sexualité dans la majorité des couples depuis le diagnostic. Seulement $\mathbf{4 0} \%$ des patients souffrant de difficultés sexuelles les reliaient à l'infertilité et $50 \%$ ne faisaient aucun lien entre les difficultés psychologiques liées à l'infertilité et les difficultés sexuelles. Ni la perte de spontanéité lors des rapports sexuels, ni les examens paracliniques jalonnant le parcours d'AMP n'influait sur la sexualité. $\mathbf{8 9 , 8 \%}$ de nos patients sont prêts à modifier leur sexualité pour être plus fertiles sans que cela entraîne de problème sexuel ou psychologique et le souci d'efficacité procréatrice lors des rapports sexuels n'existe que dans un tiers des cas. Nous avons relevé une dissociation sexualité/reproduction plus marquée chez les oligo-asthénospermes que chez les azoospermes. Pour finir, $28,2 \%$ de nos patients sont soulagés lorsqu'ils parlent de leurs problèmes psychologiques et/ou sexuels avec un psychiatre.

Conclusion : Nous avons relevé de nombreuses contradictions et sous-estimations dans les répon-

\section{Correspondance :}

Dr Michel Bellone - 22 avenue Timon David, 13013 Marseille - Tel 04.42.16.18.74 - Fax 04.42.16.18.75 Emailm.bellone@free.fr 
ses. Nous avons confirmé l'existence d'une souffrance psychologique et sexuelle mais moins importante quantitativement que celle décrite dans les populations " gynécologiques ». Quoiqu'il en soit, il existe bien une réelle souffrance psychologique et sexuelle dans notre population. C'est pourquoi nous proposons d'emblée une prise en charge préventive psychosomatique et sexologique de l'infertilité dans laquelle le praticien, qu'il soit andrologue ou psychiatre, devra aider le patient à verbaliser les affects inhérents à cette " nouvelle sexualité médicalisée ».

Mots-clés : sexualité, stérilité, andrologie, psychiatrie de liaison

\section{INTRODUCTION}

L'hypertechnicité de l'Assistance Médicale à la Procréation (AMP) a bouleversé nos conceptions de la procréation et de la sexualité. L'infertilité est désormais traitée comme une maladie incontestablement reliée à la sexualité. Nombre d'aspects sont remis en causes : éthiques, médicaux, juridiques, sociologiques et philosophiques. Les hommes et leur couple face au diagnostic d'infertilité sont le plus souvent en grande souffrance d'autant que la prise en charge médicale de ce trouble est parsemée d'embûches. De plus elle ne répond pas à toutes les questions individuelles et conjugales qui se posent, même si elle parvient, au final, à la naissance de l'enfant. Ces hommes et leur couple sont également atteints dans leur sexualité, parce qu'elle est intrinsèquement en communication au sein d'un couple amoureux et désirant et parce qu'elle se trouve déviée de sa pleine signification - voire vidée de son sens - ou exclue lors de la prise en charge. Aborder les interactions entre la sexualité et l'AMP présenterait donc un intérêt tout particulier pour suivre et aider nos patients. C'est dans cette perspective que nous avons entrepris une étude s'intéressant au retentissement sexuel, conjugal et social de l'infertilité au sein d'une population andrologique consultant pour stérilité, replaçant ainsi l'homme stérile dans son contexte biopsychosocial. Cette étude s'est faite conjointement avec un travail étudiant les mécanismes de défense psychologique dans ce même type de population afin de mieux décrire le contexte clinique qui entoure la prise en charge de ces hommes.

\section{OBJECTIFS}

Nombre d'études de la littérature $[3,11,12,13,23,25]$ avancent le fait que l'infertilité occasionne un retentissement psychologique, social et sexuel majeur pour le cou- ple. Dans la majorité des cas, ces études sont effectuées dans les services de gynécologie et sont plutôt centrées sur les retentissements féminins et conjugaux de cette infertilité. Il n'existe pas, à notre connaissance, de travaux étudiant spécifiquement ces troubles en milieu andrologique. Après avoir décrit le contexte clinique qui entoure cette infertilité à l'aide d'un "Questionnaire Général sur l'infertilité masculine $(Q G) »$, nous avons étudié chez nos patients à l'aide du "Questionnaire sur le retentissement sexuel, conjugal et social de l'infertilité chez l'homme $(Q S)$ » cinq grands axes : l'exploration du retentissement psychosocial, conjugal et sexuel de l'infertilité masculine ; la représentation du psychiatre dans la prise en charge de ces troubles sexuels et/ou psychologiques ; l'évaluation de la pertinence du questionnaire.

\section{MATERIEL ET METHODES}

\section{Population}

L'échantillon étudié comportait 39 sujets divisés en deux groupes : 21 patients azoospermes et 18 patients oligoasthénospermes (OATS). Nos patients étaient âgés de 32 ans en moyenne (ET : 5,3 ) mariés pour $79,5 \%$ et la moitié $(52,5 \%)$ n'avait pas le baccalauréat. Ces sujets ont été recrutés à la consultation d'andrologie du CHRU de Lille selon leur ordre d'arrivée pendant une période de 6 mois. Il n'y avait aucune différence significative pour l'âge et le niveau d'étude entre les 2 groupes.

\section{Critères d'inclusion et d'exclusion}

Les critères d'inclusion étaient médico-somatiques et nos sujets répartis en deux groupes : azoospermie et oligoasthénospermie. Dans le groupe d'oligo-asthénospermes, les formes sévères -moins de 1 million de spermatozoïdesétaient majoritaires.

Les critères d'exclusion étaient : les maladies chroniques graves, engageant le pronostic vital à moyen et à long terme ; les troubles neurologiques graves ; les anomalies chromosomiques et les maladies génétiques ; les troubles sexuels à l'origine de l'infertilité ; les demandes de reperméabilisation des canaux déférents post-vasectomies et les patients maîtrisant mal la langue française ou illettrés ou présentant un niveau intellectuel liminaire.

\section{Instruments cliniques}

\section{a)Le Questionnaire Général sur l'infertilité masculine $(Q G)$}

Nous avons réalisé, au sein des services d'Andrologie et de Psychiatrie de Liaison du CHRU de Lille, un questionnaire général sur l'infertilité correspondant à un entretien semistructuré comportant 40 questions s'inspirant des constata- 
tions cliniques. Il permet d'apprécier le contexte symptomatique et clinique dans lequel évolue ces hommes. Il se passe en un temps moyen de 10 minutes et permet d'explorer, chez l'homme infertile : des éléments biographiques personnels et familiaux, ses antécédents médico-chirurgicaux et psychiatriques, son état de santé actuel, le type d'infertilité, la spécialité du médecin qui lui a appris le diagnostic et pour le couple : l'historique du couple et de la maladie, la technique d'AMP adoptée, etc...

\section{b) Le questionnaire sur le retentissement sexuel, conju- gal et social de l'infertilité chez l'homme (QS)}

Il s'agit d'un autoquestionnaire que nous avons réalisé au sein des services d'Andrologie et de Psychiatrie de Liaison du CHRU de Lille comprenant 50 questions, inspiré de constatations cliniques et de données de la littérature [13, $22,25,26,29,30]$. Il a une valeur exploratoire et descriptive pure. Il se passe en 40 minutes et permet :

- d'apprécier le retentissement social, psychologique et sexuel de l'infertilité pour l'homme et pour le couple ;

- de rechercher des troubles de la sexualité chez l'homme avant puis après l'annonce du diagnostic d'infertilité ;

- l'évaluation de la sexualité de l'homme et du couple à un niveau quantitatif, qualitatif et en termes de satisfaction sexuelle ;

- d'évaluer la relation de couple confrontée au problème de l'infertilité ;

- de rechercher chez les hommes et au sein du couple, un lien de cause à effet entre l'infertilité et ses différentes répercutions psychologiques et/ou sexuelles ;

- l'évaluation du retentissement des examens complémentaires et des traitements sur la sexualité des hommes infertiles et du couple ;

- d'apprécier la position impartie au psychiatre quant à la prise en charge d'un éventuel trouble sexuel et/ou psychologique chez l'homme ;

- l'évaluation du retentissement sexuel et/ou psychologique d'un ajustement de la sexualité afin d'augmenter la fertilité du couple ;

- l'évaluation (subjective) par l'homme du retentissement de son infertilité sur sa conjointe (victime d'infertilité secondaire).

L'analyse statistique des données a été réalisée à l'aide du logiciel S.A.S version 8 au laboratoire de Biostatistiques du CHRU de Lille.

\section{RESULTATS}

\section{1. Évaluation du retentissement psychosocial de l'in- fertilité masculine}

Nous confirmons dans cette étude, que l'infertilité masculine représente un véritable « coup de poignard » narcissique en atteignant nos patients au plus profond de leur imago masculin. Pour $46,2 \%$ de nos patients, " un homme présentant des troubles de la fertilité peut avoir le sentiment d'être atteint dans sa virilité » ; par contre, ils relient peu « infertilité, virilité et puissance sexuelle » $(12,8 \%)$. Seulement $28,2 \%$ d'entre eux estiment que «l'infertilité peut entraîner un retentissement négatif majeur dans la vie sociale ", mais ils sont quand même près de la moitié à penser « qu'il est préférable d'éviter de l'annoncer à son entourage » $(46,2 \%)$. Ce qui n'empêche pas qu'une large majorité en aient parlé à leur entourage $(77 \%)$, contre $23 \%$ qui ne l'ont jamais fait. On ne peut donc s'empêcher d'évoquer ici, l'inévitable influence de la partenaire. Les deux groupes en parlent avec la même fréquence. Parmi ceux qui en parlent, la totalité l'ont fait avec leur famille, $46,7 \%$ avec leurs amis et $20 \%$ au travail.

$\mathrm{Au}$ tout début de leur prise en charge médicale, ces hommes ont surtout envisagé des problèmes conjugaux $(48,7 \%)$, psychologiques et sociaux $(23 \%)$, mais très peu dans leur sexualité $(7,7 \%)$. Étonnamment, $38,5 \%$ des hommes n'ont pas envisagé de problème ! Les difficultés inhérentes au parcours d'AMP ne semblent anticipées que de façon modérée. Le retentissement psychologique et/ou sexuel de l'infertilité masculine, est perçu - par nos patients - comme plus perturbant pour la femme que pour l'homme $(35,9 \%)$ contre $20,52 \%$ qui pensent que l'homme est plus perturbé. Dans la majorité des cas, ils ne font aucune différence de retentissement quel que soit le sexe $(43,6 \%)$. Pour la plupart de nos patients, aucun problème quel qu'il soit - n'est survenu tout au long du parcours d'AMP $(43,6 \%)$ !? Lorsqu'il y a eu problèmes (sexuel et/ou social et/ou psychologique et/ou conjugal), 25,6\% sont survenus « initialement à l'annonce de l'infertilité » ou « de façon permanente » (à tous les stades de l'AMP) et très peu $(5 \%)$ lors de la réalisation des examens médicaux ou au moment des traitements. Pour finir, prés d'un tiers $(30 \%)$ de nos patients se sont vus annoncer pour la première fois leur diagnostic lors de la remise de leur spermogramme (soit au laboratoire de biologie, soit par voie postale).

Le diagnostic est accusé à son annonce, soit comme un « coup de masse » $(39,5 \%)$, soit avec « un sentiment de déprime passagère $\gg(18,4 \%)$ et $10,5 \%$ des patients signalent « ne rien avoir ressenti sur le coup». C'est « 
l'espoir » qui revient le plus fréquemment au moment de la mise en route des traitements $(47,2 \%)$, puis la notion de «soulagement » $(33,3 \%)$ ou «d'angoisse » $(11,1 \%)$.

\section{Evaluation du retentissement conjugal de l'inferti- lité masculine}

Nos couples se connaissaient en moyenne depuis 8 ans $(E T: 3,44)$ et l'entente conjugale - pendant les démarches en AMP - était jugée comme « excellente » $(64,1 \%)$ ou «bonne » $(30,8 \%)$. La plupart de nos sujets $(82 \%)$ se représentaient leur partenaire sans difficulté sexuelle en rapport à leur diagnostic d'infertilité contre $10,3 \%$ avec et $7,7 \%$ ne sachant pas. Prés de la moitié $(48,7 \%)$ de nos sujets se représentaient leur partenaire sans difficulté psychologique en rapport avec leur diagnostic, contre $35,9 \%$ avec, et $15,4 \%$ ne sachant pas. Il existait, à ce sujet, une différence statistiquement significative entre les deux populations $(\mathrm{p}=0.0013)$. Le groupe OATS se représentait significativement plus « de difficultés psychologiques » chez la partenaire que le groupe azoosperme.

Nombre $(41 \%)$ de nos sujets éprouvaient un sentiment de culpabilité envers leur partenaire en rapport avec leur diagnostic d'infertilité, mais $54 \%$ n'en éprouvaient pas et $5 \%$ ne savaient pas. Les difficultés rencontrées dans le couple étaient perçues comme : secondaires à des troubles psychologiques en rapport avec l'infertilité $(15,4 \%)$, conséquences directes de l'infertilité à l'origine de problèmes de couple $(12,8 \%)$, liées à l'interruption des rapports devant l'impossibilité de grossesse $(7,7 \%)$, la perte du désir érotique effacé par le seul désir de grossesse (5\%), l'interdiction inconsciente du plaisir pour montrer le désir d'enfant (5\%). Mais, dans $1 / 3$ des cas aucune raison n'était avancée pour expliquer ces difficultés. Les difficultés conjugales duraient en moyenne depuis 3,14 ans (ET : 3 ans).

\section{Evaluation du Retentissement sexuel de l'infertili- té masculine}

La fréquence des rapports sexuels dans notre population était - en moyenne - de 2,5 rapports par semaine (ET : 1,3). La majorité de nos patients $(64,1 \%)$ étaient satisfaits de cette fréquence mais $35,9 \%$ ne l'étaient pas et en demandaient plus à $100 \%$. Nous avions $10,3 \%$ de troubles sexuels avant le diagnostic d'infertilité masculine contre $25,6 \%$ lors de la réalisation de notre étude. Lorsque ces troubles survenaient après le diagnostic, ils se répétaient plus fréquemment. Parmi les patients qui avaient des difficultés sexuelles, seulement $40 \%$ faisaient un lien entre infertilité et difficultés sexuelles contre $30 \%$ qui n'en faisaient pas. La moitié $(50 \%)$ ne faisaient aucun lien entre difficultés psychologiques et difficultés sexuelles contre $40 \%$ qui en faisaient un. Contrairement aux données de la littérature $[13,20,25,27]$, la perte de spontanéité lors des rapports sexuels - rapports périovulatoires « obligatoires » - n'influait pas sur la sexualité et les examens paracliniques jalonnant le parcours d'AMP ne la modifiaient pas non plus dans $87,3 \%$, et lorsqu'ils la modifiaient $(12,8 \%)$, ils la dégradaient dans tous les cas.

A une écrasante majorité, nos patients $(89,8 \%)$ sont prêts à modifier leur sexualité pour être plus fertiles. Tous les OATS le sont, alors que ce n'est pas le cas pour les azoospermes, sans qu'il y ait toutefois de différence significative. Cette modification n'entraînait, dans $92,3 \%$ des cas, ni de problèmes sexuels, ni de problèmes psychologiques, contrairement aux données de la littérature $[13,14,17,18$, $19,25]$. Lorsqu'elle en entraînait $(7,7 \%)$, ces problèmes étaient toujours considérés comme peu importants. Nos patients n'avaient un «souci d'efficacité procréatrice » lors des rapports sexuels que dans $34,3 \%$ des cas (Tableau $1)$.

Tableau 1 : Souci d'efficacité procréatrice lors des rapports sexuels dans notre échantillon d'hommes stériles.

\begin{tabular}{llll}
\hline $\begin{array}{l}\text { Souci d'efficacité } \\
\text { procréatrice lors des } \\
\text { rapports sexuels }\end{array}$ & $\begin{array}{l}\text { Echantillon } \\
\text { Total }\end{array}$ & Azoospermes & OATS \\
\hdashline Oui & $34,3 \%$ & $28,6 \%$ & $41,2 \%$ \\
Non & $63,1 \%$ & $71,4 \%$ & $53,0 \%$ \\
Ne Sait Pas & $2,5 \%$ & $0 \%$ & $5,8 \%$ \\
$\begin{array}{l}\text { Nombre total de } \\
\text { répondants }\end{array}$ & 38 & 21 & 17 \\
\hline
\end{tabular}

En ce qui concerne le vécu par nos patients de leur sexualité conjugale : il n'y a pas eu de modification de la sexualité dans la majorité des couples depuis le diagnostic $(61,5 \%)$. Lorsque celle ci se modifiait on notait : une diminution de la fréquence des rapports dans la majorité des cas $(30,7 \%)$, une stimulation avec augmentation de la fréquence des rapports $(7,7 \%)$, une détérioration de la « qualité » des rapports $(7,7 \%)$. On a pu, aussi, noter que la plupart de nos couples ne présentaient aucune détérioration de leur sexualité $(74,4 \%)$. Lorsqu'il y avait difficultés sexuelles dans le couple - 25,6\% de l'effectif total - elles duraient en moyenne depuis 2,2 ans (ET:2) et l'on retrouvait : une diminution de la fréquence des rapports sexuels (23\%), une sensation de sexualité insatisfaisante ou une disparition de la fantaisie pendant les rapports (5\%).

4. Représentation du psychiatre dans la prise en charge des problèmes psychologiques et sexuels liés à l'infertilité masculine 
$28,2 \%$ de nos patients sont soulagés lorsqu'ils parlent de leurs problèmes psychologiques et/ou sexuels avec un psychiatre. $53,9 \%$ des patients n'ont pas plus de problème à parler de leur sexualité à un médecin plutôt qu'à un autre (psychiatre ou pas). 10,2\% sont heurtés (gênés) de parler de leur sexualité quel que soit le type de médecin et $17,9 \%$ lorsque c'est un psychiatre. En conséquence, 7,7\% des patients sont spécifiquement dérangés lorsqu'il s'agit d'un psychiatre, à savoir qu'ils peuvent parler de leur sexualité à un médecin «somaticien » mais pas à un psychiatre. Nous nous attendions à des chiffres bien plus élevés pour une population n'ayant pas l'habitude de consulter en psychiatrie. Pour finir, $82 \%$ des patients n'ont pas de gêne particulière à aborder leur sexualité avec un psychiatre.

\section{Evaluation de la pertinence et de la qualité du questionnaire pour les hommes interrogés}

La majorité des patients n'ont pas de remarque à formuler sur le questionnaire $(84,6 \%)$ et pour de nombreux sujets, il aide à la réflexion. Il n'y a pas eu de difficulté lors de la réponse à ce questionnaire $(87 \%)$.

\section{DISCUSSION}

Signalons tout d'abord l'absence d'études similaires effectuées en population andrologique ce qui nous empêche de pouvoir comparer nos résultats. Nous n'avons qu'artificiellement séparé le retentissement conjugal du retentissement sexuel tout en sachant qu'il sont indissociables.

Un des objectifs de ce questionnaire était d'apprécier comment les patients d'un service d'andrologie, ne consultant habituellement pas en psychiatrie, appréhendaient ou pas une consultation psychiatrique, afin d'aborder leurs problèmes psychologiques et/ou leur sexualité. Nous devons dans un tel service explorer davantage le versant psychique de cette sexualité et son intrication avec le désir d'enfants. $28,2 \%$ des patients sont soulagés lorsqu'ils abordent leurs problèmes psychologiques et/ou sexuels. Les troubles sexuels sont une souffrance : en parler soulage. Ce seul chiffre justifie à lui seul qu'un professionnel connaissant parfaitement les intrications entre psychisme, sexualité et AMP propose un espace de paroles à ces patients. Nous sommes probablement avec ce chiffre, comme dans tous les questionnaires ayant trait à la sexualité $[1,4,9]$, en deçà du chiffre réel ; certains avouent qu'ils seraient soulagés $(28,2 \%)$, peut-être il y en aurait-il plus ? Une large majorité de sujets n'ont «pas de gêne particulière » $(82 \%)$, ni de "problèmes particuliers » à parler de sexualité avec un psychiatre. Est-ce une réponse normative ? N'osent-ils pas avouer leur sentiment de gêne ou de soulagement ? Face à l'hypertechnicité et à la demande "d'agir » en AMP, le temps de la parole pourrait être occulté. Selon Rossin-Amar «Si la parole est gommée, la symbolique l'est aussi et il ne reste plus que des corps machines et des techniciens. Les variations de sexualité n'y sont que des indicateurs d'une autre parole qu'il faut savoir décoder " [30] ; restaurons donc cette parole avant que ces indicateurs se manifestent.

Nous avons relevé une discordance des réponses en ce qui concerne la satisfaction sexuelle : une faible proportion d'hommes $(10,25 \%)$ jugent d'emblée leur sexualité insatisfaisante. Notons que la notion de « sexualité plutôt satisfaisante » retrouvée dans la littérature [4] est peu éloquente, Brenot [9] nous dit que «dans ce secteur de la vie qui cultive l'absolu, on ne peut être que très satisfait ou pas du tout ». Nos patients «plutôt satisfaits » $(61,55 \%)$ pencheraient peut-être vers l'insatisfaction sans que nous puissions réellement l'affirmer. 25,6\% de nos patients jugeaient leur sexualité "détériorée ». Pour finir, 35,9\% des hommes sont insatisfaits de la fréquence des rapports sexuels. Nous constatons donc que le nombre d'insatisfaits estimé directement à la question "comment jugez-vous votre sexualité ? " était en fait sous-déclaré $(10,25 \%)$. Le nombre réel d'insatisfaits au niveau de la sexualité se révèle être plus important mais nos sujets n'osent pas se l'avouer d'emblée. Différents croisements statistiques nous ont permis de montrer que tous les sujets qui se déclarent « insatisfaits » ont des troubles sexuels ; par contre, parmi ceux atteints des troubles sexuels, $50 \%$ considèrent leur sexualité comme "plutôt satisfaisante 》 et $10 \%$ "très satisfaisante ». Nous évoquons donc un possible hiatus entre les troubles organiques sexuels et leur perception subjective.

L'inscription comme « infertile » et l'entrée dans le circuit d'AMP perturbent la sexualité avec des troubles plus nombreux et plus répétitifs $[15,20,26,30]$. Après l'annonce de l'infertilité, le pourcentage de troubles sexuels augmentait de $15,3 \%$. Sur un plan qualitatif, on note, dans notre population, plus particulièrement un accroissement des troubles du désir évoquant une sexualité désérotisée. L'ensemble des données de la littérature $[13,25,26,28]$ s'accordent à dire - même si leurs méthodologies et populations sont différentes de la nôtre - que la prévalence des troubles sexuels s'accroît nettement après le diagnostic d'infertilité. Cet accroissement ne nous surprend pas ; en effet l'instinct de survie d'une espèce associe la sexualité à la reproduction. Si la reproduction est menacée, il n'y a rien d'étonnant à ce que la sexualité chez l'homme et sa partenaire s'en ressente. Lachcar propose [23] d'emblée une prise en charge psychosomatique de l'infertilité pour justement prévenir l'apparition de ces difficultés sexuelles secondaires. Par contre, nous aurions pu nous attendre, chez nos patients, à des troubles sexuels au moins plus fréquents qu'en population générale (Tableau 2), ce qui n'est pas le cas. Giuliano et al., [21] nous disent qu'un tiers de la population française présente une insuffisance érectile, nous 
Tableau 2 : Troubles sexuels après le diagnostic dans notre échantillon d'hommes stériles versus troubles sexuels en population générale.

\begin{tabular}{lllll}
\hline $\begin{array}{l}\text { Troubles sexuels dans la } \\
\text { population française [1] }\end{array}$ & Souvent (\%) & Parfois (\%) & $\begin{array}{l}\text { Troubles sexuels } \\
\text { dans notre population } \\
\text { après le diagnostic (\%) }\end{array}$ & $\begin{array}{l}\text { Troubles sexuels } \\
\text { correspondants dans } \\
\text { notre questionnaire }\end{array}$ \\
\hline $\begin{array}{l}\text { Absence totale d'érection } \\
\text { Éjaculation précoce }\end{array}$ & 7 & 12 & 2,6 & Dysérection \\
Anéjaculation & 10 & 27 & 7,7 & Éjaculation précoce \\
Anorgasmie & 2 & 5 & 7,7 & Anéjaculation \\
Absence ou insuffísance de désir sexuel & 3 & 7 & 0 & Anorgasmie \\
& & 16 & $7,7+10,3=18,0$ & Absence de désir et baisse \\
de la libido
\end{tabular}

n'en avions que $2,6 \%$ dans notre population. La sousdéclaration des troubles sexuels est quasi certaine dans notre population. Nous émettons plusieurs hypothèses pour expliquer ce phénomène : souffrance, déjà suffisante, d'être infertile? Biais méthodologique (questionnaire et/ou examinateur) ? Mécanismes de défense ne permettant pas l'expression de ces troubles? Déni ?

Nos sujets OATS se représentaient, significativement plus, leur partenaire avec des troubles psychologiques en rapport avec l'infertilité masculine que les azoospermes. Nous émettons l'hypothèse que cela pourrait s'expliquer par le fait que les partenaires d'azoospermes se sentent complètement dédouanées de toute responsabilité dans l'infertilité ; par conséquent la situation est plus claire, moins anxiogène, le deuil se ferait mieux, l'homme porterait toute la responsabilité de l'infertilité. Alors que chez les OATS il reste une possibilité d'infertilité "mixte », la situation est alors moins claire et plus anxiogène pour la partenaire chez qui un doute, à l'origine de troubles psychologiques, pourrait survenir. $90,5 \%$ des azoospermes pensent que leurs partenaires ne présentent pas de troubles psychologiques ; on peut encore s'étonner devant une telle dénégation et évoquer une probable sous déclaration ou pour le moins, une sous-estimation de la part des hommes. Cela dit, pour étayer ces différentes hypothèses, il faudrait faire une étude, avec des diagnostics type DSM-IV [2], afin d'apprécier la prévalence des troubles psychiatriques chez les partenaires d'OATS et chez les partenaires d'azoospermes. De même, $82 \%$ des hommes n'envisageaient pas de troubles sexuels chez leur partenaire! Bien que nous ne connaissions pas la prévalence des troubles sexuels chez les conjoints de nos sujets, les données de la littérature en population gynécologique $[13,14,20,25,26,28]$ nous laissent penser qu'il y a - ici encore - une possible sousestimation de la part des hommes.

Comme on peut aussi le constater en clinique, les hommes azoospermes ont beaucoup moins " de soucis en termes de reproduction dans leurs rapports sexuels » lors du coït que les hommes OATS (28,6 contre 41,2\%) (Tableau 1) sans qu'il y ait de différence significative. Nous émettons l'hypothèse que les azoospermes et/ou leur partenaire auraient fait le deuil de la reproduction par voie naturelle. Ainsi, dans leurs rapports sexuels, ils laisseraient de côté la fonction reproductrice. Ce qui ne serait pas le cas des sujets OATS et/ou de leur partenaire qui s'efforceraient de « faire au mieux ». Les différents croisements statistiques - effectués dans notre étude - montrent que la « diminution de la fréquence des rapports sexuels " aurait tendance à moins déranger les azoospermes que les OATS et leur qualité de vie sexuelle serait un peu mieux préservée. Les azoospermes ont moins de rapports, mais ils ont une "qualité de vie sexuelle » mieux préservée que les OATS. Les azoospermes dissocieraient sexualité et reproduction alors que ce ne serait pas le cas pour les OATS. La minorité d'OATS ayant une «diminution de la fréquence des rapports » l'associent dans les $2 / 3$ des cas à " une détérioration de la qualité des rapports ». Les azoospermes eux, présentent plus de « diminution de la fréquence des rapports » sans l'associer pour autant à une « une détérioration de la qualité des rapports ». D' autres réponses confirment cette tendance : seuls les OATS notent une « stimulation avec augmentation de la fréquence des rapports » alors que la « diminution de fréquence des rapports " concerne surtout les azoospermes qui sont aussi les seuls à répondre «les rapports sont inutiles car il n'y a pas de grossesse possible ». Tous les OATS sont «prêts à modifier leur sexualité pour être plus fertiles », ce qui n'est pas le cas des azoospermes. Enfin, il n'y a que des azoospermes qui malgré le signalement d'une « diminution de fréquence des rapports » sont satisfaits de leur sexualité. Il ne faut pas oublier l'influence de la partenaire qui selon nous « stimulerait » lorsqu'il y aurait une possibilité de reproduction par voie naturelle et qui semblerait moins motivée dans le cas contraire. Nous notons aussi une curieuse dissociation vie sexuellevie conjugale, puisque $95 \%$ des patients qui envisagent des problèmes « au tout début de leur prise en charge » dans leur vie conjugale à venir n'en imaginent pas dans leur vie sexuelle ? Il n'y a pas de différence entre les deux groupes à ce niveau. Notre étude révèle que les examens cliniques 
et paracliniques du parcours d'AMP n'ont pas d'influence sur la sexualité de nos sujets, ce qui va à l'encontre des données de la littérature $[13,15,25,28]$.

Nous nous interrogeons sur ces $38,5 \%$ d'hommes qui "n'ont envisagé aucun problème au tout début de la prise en charge pour infertilité » et sur ces $43,6 \%$ d'hommes pour lesquels «aucun problème n'est survenu »!? Ce qui là, encore, va à l'encontre, non seulement des données de la littérature, mais des données cliniques. Sommes-nous dans le déni ? Peut-on parler de déni quand nous avons $41 \%$ des hommes qui culpabilisent vis-à-vis de leur partenaire ? Certains auteurs remarquent que lorsque l'homme se croit infertile, il peut avoir divers comportements. Il peut par une attitude de déni éviter d'aborder réellement le problème. Cela peut être camouflé derrière un activisme professionnel ou sportif mis en place pour effacer et nier l'événement en remettant toujours à plus tard la prise en compte de ce problème $[5,19,26,27]$. Cependant, nous avons étudié les modalités défensives dans cette population à l'aide d'une échelle psychométrique validée : le Défense Style Questionnaire (DSQ) [8] et ni le déni, ni l'activisme ne sont utilisés significativement par cette population [6]. Nous pourrions peut-être plus évoquer - dans ce cas - la dénégation. En effet, Il convient de bien différencier le déni de la dénégation : la dénégation est un procédé par lequel le sujet, tout en formulant un de ses désirs, pensées, sentiments jusqu'ici refoulés, continue à s'en défendre en niant qu'il lui appartienne [24] ; elle permet d'éliminer une représentation gênante en refusant de la reconnaître comme nous concernant, alors que le déni nie la réalité même [7]. Cette dénégation expliquerait peut-être ces $66,6 \%$ de patients s'opposant au groupe de paroles proposé dans le QS. Il y a donc nécessité de travailler pour aider ces hommes à médiatiser leur souffrance - par un autre vecteur que le groupe de paroles - afin de prévenir l'émergence de troubles psychiatriques surreprésentés dans cette population [6]. Cela pourrait être une psychothérapie de soutien pour les sujets dépistés comme à « risque » par l'andrologue ou proposée de façon systématique. Comme pour les populations gynécologiques, il n'y a pas de consensus sur la présentation la plus appropriée de ce soutient médico-psychologique au patient. Il nous semble cependant, que pour garantir un taux de réussite maximal, elle doit passer par l'andrologue.

Notre étude nous apprend avec effroi, qu'un tiers de nos patients apprennent leur terrible diagnostic via la remise de leur spermogramme soit par le biologiste directement, soit par la poste. Ils sont alors seuls, confrontés à ce terrible verdict, face à la froideur d'une feuille de papier. Nous ne pouvons discuter un tel résultat, si ce n'est de faire en sorte que ce chiffre baisse au plus vite et de s'interroger sur une éventuelle formation psychologique des biologistes face aux résultats qu'ils peuvent distribuer sous plis. Les résul- tats du spermogramme sont souvent commentés de manière maladroite, y compris dans les comptes rendus rédigés par les biologistes sur le spermogramme lui-même avec de tels commentaires : "sperme insuffisant, pauvre, spermatozoïdes paresseux, malformés, morts... ». À chaque commentaire d'examen, la partie témoigne du tout et l'individu s'identifie à ses spermatozoïdes, le blessant encore plus dans son narcissisme et augmentant sa culpabilité.

Les conjointes de nos patients, à $90 \%$, ne se sentent pas frustrées par l'abord surtout masculin de l'infertilité dans notre étude (QG). Pourtant, elles sont victimes d'une " infertilité secondaire" et vont mettre en place des mécanismes de défense pour se protéger de cette maladie «qui $n$ 'est pas la leur ». Briguet-Lamarre nous dit que « cet arrêt imposé et brutal dans l'accomplissement projeté de leur féminité va susciter des réactions très différentes selon le niveau de maturité de chacune et selon la relation du couple préexistante »[10]. Souvent les hommes souffrent seuls avec une stérilité qui est parfois vécue avec agressivité par la femme "privée du droit à l'enfant par la faute de son partenaire » [10]. Nous n'avons pas retrouvé - ni en pratique clinique, ni dans notre étude - une telle agressivité chez les partenaires de nos patients.

Le QS se terminait par une série de questions ouvertes sur son intelligibilité et sur sa perception. A priori le questionnaire a bien été compris et il n'était pas perçu comme « agressif » par nos patients. Nous avons constaté qu'il constituait même pour nombre d'entre eux un point de départ à la réflexion. Dans tous les cas, il a constitué pour nous une base clinique permettant d'aborder avec le patient certains problèmes, voire certains conflits : ce questionnaire les aidant à mieux élaborer et verbaliser leur problématique et les conflits psychiques inhérents à la l'AMP. Il nous a permis de mieux connaître l'état de souffrance sexologique et psychologique de notre population afin de permettre une prise en charge et un accompagnement psychiatrique ou médico-psychologique plus pertinent et prévenir la survenue de complications psychiatriques ce qui, en soit, est un objectif majeur de la psychiatrie de liaison [16].

\section{CONCLUSION}

Ce travail nous a permis d'évaluer dans une population spécifiquement andrologique le retentissement psychologique, social et sexuel de l'infertilité. Nous avons relevé, comme dans la majorité des travaux ayant trait à la sexualité $[1,4,9]$, de nombreuses contradictions et sous-estimations dans les réponses. Nous avons confirmé l'existence d'une souffrance psychologique et sexuelle mais moins importante quantitativement que celles décrites dans les populations " gynécologiques ». Quoiqu'il en soit il existe bien une réelle souffrance 
psychologique et sexuelle dans notre population. Nous avons relevé une dissociation sexualité/reproduction plus marquée chez les OATS que chez les azoospermes. Ainsi qu'une recrudescence des troubles sexuels à la suite du diagnostic et l'entrée dans le parcours d'AMP. Cependant et contrairement aux données de la littérature, la sexualité n'est pas perturbée par les différents examens. Nous proposons d'emblée, comme Lachcar [23], une prise en charge psychosomatique et sexologique de l'infertilité pour justement prévenir l'apparition de ces difficultés sexuelles. D'autre part, l'infertilité peut venir révéler l'existence d'une problématique sexologique conjugale masquée par la demande d'enfant. Dans ce cas aussi l'aide thérapeutique de type sexologique et psychologique pourra être souhaitable.

Notre questionnaire a constitué une base clinique en contribuant à l'élaboration des difficultés psychologiques et sexuelles en rapport avec l'infertilité et son traitement. Il a été abordé par nos patients, à l'occasion de ce questionnaire, des thèmes parfois tabous - difficultés psychologiques, sexuelles et conjugales, onanisme (plus clandestin que jamais !), velléités d'infidélité, etc... - qui n'avaient jamais été évoqués tout au long de cet épuisant parcours d'AMP. Nous savons que - pour près d'un tiers de notre population - aborder les troubles sexuels avec un médecin (psychiatre ou pas) provoque un sentiment de soulagement. Il est donc souhaitable que le praticien - qu'il soit psychiatre ou andrologue - aide le patient à verbaliser les affects inhérents à cette « nouvelle sexualité médicalisée ». Rossin-Amar rappelle la nécessaire humilité face aux taux important d'échecs en AMP qui nous renvoie à la place inévitable du désir et de son expression. Il est fondamental que le médecin restaure l'homme infertile à la place de celui au Nom de qui se fait l'AMP, position symbolique qui permet, entre autre, de conserver l'équilibre sexuel et relationnel du couple [30] et de ne pas enfermer le patient au rôle d'objet apprécié sur le seul critère de son sperme.

\section{RÉFÉRENCES}

1. ACSF INVESTIGATORS : AIDS and sexual behaviour in France. Nature, 1992, $360: 407-409$.

2. AMERICAN PSYCHIATRIC ASSOCIATION : Manuel Diagnostique et Statistique des Troubles Mentaux (DSM-IV). Washington, 1994. Traduction française : GUELFI J.D. et al., Paris, Masson, 1996.

3. ATHEA N. : La stérilité : une entité mal définie. In : Testart J. ed. Le magasin des enfants. Paris, Folio actuel, 1994.

4. BAILLY T. : Les évaluations psychométriques en Sexologie, revue bibliographique. Thèse pour le Doctorat d'Etat en Médecine, Médecine Spécialisée Clinique, 1997, Bordeaux II, N³065.

5. BEETSCHEN A. : Vouloir un enfant, devenir père. Entrevue, $1981,1: 28-30$.

6. BELLONE M. : Modalités défensives utilisées dans une population d'hommes consultant pour stérilité : évaluation par le DSQ, étude des troubles psychiatriques et sexuels. Mémoire pour le D.E.S. de psychiatrie Inter-Région Nord-Ouest, 2001, Lille, $\mathrm{N}^{\circ}$ 331157-1157.

7. BERGERET J. : Problème des défenses. In : Psychologie Pathologique. Paris, Masson, $6^{\circ}$ édition, 1972/1995: 90-112.

8. BOND M., GARDNER S.T., CHRISTIAN J. et al. : Empirical study of self-rated defense styles. Arch. Gen. Psychiatry, 1983, $40: 333-338$.

9. BRENOT P. : Inventer le couple. Paris, Odile Jacob, 2001.

10. BRIGUET-LAMARRE M. : Aspects psychologiques de la stérilité masculine irréversible, de ses incidences chez la femme et de la demande de procréation du couple par insémination artificielle. Rev. Franç. Gynéc., 1980, 75 : 553-556

11. BYDLOWSKI M., DAYAN-LINTZER M., CAHEN F. et al. : L'Approche psycho-médicale de l'infertilité. J. Gyn. Obstet. Biol. Reprod., 1983, 12 : 269-276.

12. BYDLOWSKI M., DAYAN-LINTZER M. : Désir d'enfant, mal d'enfant. Psychosomatique, 1985, $1: 25-42$.

13. CHAMPS F. : Troubles psychologiques et sexuels secondaires au diagnostic et au traitement de l'infertilité. Thèse pour le Doctorat d'État en Médecine, Médecine Spécialisée Clinique, Rennes, $1997, \mathrm{~N}^{\circ} 128$

14. CHATEL M.M. : Le désir escamoté. In : Testart J. ed. Le magasin des enfants. Paris, Folio actuel, 1994 : 65-80.

15. CHATEL M.M. : Incidences des techniques modernes sur la paternité ou « on demande un enfant hors sexe ». Neuropsychiatrie de l'enfance, 1988, $36: 475-479$.

16. CONSOLI S.M. : La psychiatrie à l'hôpital général. Encycl. Méd. Chir. (Elsevier, Paris), Psychiatrie, 37-958-A-10, 1998 : 11.

17. CZYBA J.C. : L'infertilité masculine, aspects psychologiques. Soins Gyn. Obstet. Puer. Ped., 1991, 118 : 27-29.

18. CZYBA J.C. : Approche psychologique de l'homme infécond et stérile. Rev. Prat., 1993, 43 : 979-980.

19. DELAISI de PARSEVAL G. : Les PMA ou paternités médicalement assistées. In : Marciano P. ed. Le père, l'homme et le masculin en périnatalité. Ramonville, Erès (spirale), 1999, 11:111124.

20. EPELBOIN S. : Nouvelles procréations, nouvelles sexualités ? Gynécol. Psychosom., 1991, $1: 29-34$.

21. GIULIANO F., CHEVRET-MEASSON M., TSATSARIS P., et al. : Prévalence de l'insuffisance érectile en France : résultats d'une enquête épidémiologique menée auprès d'un échantillon représentatif de 1004 hommes. Prog. Urol., 2002, 12 : 260-267.

22. GUERRA D., LLOBERA A., VEIGA A. et al. : Psychiatric morbidity in couples attending a fertility service. Hum. Reprod., $1998,13: 1733-1736$.

23. LACHCAR P. : L'homme infertile : un homme en souffrance. 
Contracept. Fertil. Sex., 1992, $20: 735-736$.

24. LAPLANCHE J., PONTALIS J.B. : Vocabulaire de la psychanalyse. Paris, PUF, 1967.

25. MARDON-LEROLLE F. : Incidence de l'infertilité et des techniques d'assistance médicale à la procréation sur la sexualité des couples. Thèse pour le Doctorat d'État en Médecine, Médecine Spécialisée Clinique, Nantes, 2000, N $153 \mathrm{M}$.

26. MIMOUN S. : Les multiples interactions entre l'infertilité et la sexualité. Contracept. Fertil. Sex., 1993, 21 : 251-254.

27. PERRET Z. : Stérilité et AMP du côté des hommes. Reprod. Hum. Horm., 2000, 13 : 185-187.

28. PROUVOST F. : De la place de la technique médicale dans la sexualité du couple. Contracept. Fertil. Sex., 1989, $17: 631-633$.

29. QUINTIN A. : Désir d'enfant et désir sexuel. Cahiers Sexol. Clin., 1994, $20: 33-36$.

30. ROSSIN-AMAR B., BONIERBALE M. : Sexualité et PMA. Sexologies, 1995, $4:$ 13-15.

\section{ABSTRACT}

Sexual, marital and social effects in a population of sterile males consulting in Andrology: a liaison psychiatrist's opinion

Michel BELLONE, Jean-Marc RIGOT, Olivier COTTENCIN, Michel GOUDEMAND

Infertility is from now treated as a disease inevitably linked to sexuality. Men and couples faced with a diagnosis of infertility generally experience considerable distress. Their sexuality is also affected, as sexuality is intrinsically related to love and desire in the couple and because sexuality loses its full significance- or even becomes meaningless - or is excluded from therapy. Analysis of the interactions between sexuality and artificial insemination would be useful to help our patients. Many studies in the literature conclude that infertility causes major psychological, social and sexual effects on the couple. Most of these studies were conducted in gynaecology units and concerned the female and sexual effects of infertility. We therefore conducted a study specifically devoted to a male population consulting for sterility, placing the sterile man in his biological and psychosocial context.

Material and methods: The study population consisted of 39 subjects divided into two groups: 21 azoospermic males and 18 oligoazoospermic males. These men were selected in andrology departments over a period of six months. The andrology and liaison psychiatry departments of Lille university hospital established a general infertility questionnaire. This semi-structured questionnaire comprising $\mathbf{4 0}$ questions was based on clinical experience and describes the clinical and symptomatic context of infertility. An individual 50-item questionnaire was then used to analyse the psychosocial, marital and sexual effects of male infertility and the representation of the psychiatrist in the andrology department.

Results : One third of patients discovered the diagnosis of infertility when the biologist gave them the results of the semen analysis. Our patients initially envisaged marital, psychological and social problems, but very few sexual problems. They imagined that infertility was more disturbing for women than for men, from a psychological and sexual point of view. $82 \%$ of our subjects imagined that their partner had no sexual problems. $48.7 \%$ of them thought that their partner had no psychological difficulties related to their infertility. The oligoasthenospermia group considered that the partner had significantly more "psychological difficulties" compared to the azoospermia group. $41 \%$ of our patients felt guilty towards their partner because of their infertility diagnosis. $10.3 \%$ of patients presented sexual disorders before the diagnosis of male infertility and $25.6 \%$ presented sexual disorders after this diagnosis. Contrary to data in the literature, a major change of sexuality was not observed in the majority of the couples after the diagnosis. When sexuality changed, it generally consisted of a reduction of the frequency of sexual relations. Among the patients who reported sexual difficulties, only $\mathbf{4 0} \%$ related them to infertility. $\mathbf{5 0} \%$ did not report any relationship between the psychological difficulties related to infertility and the sexual difficulties. Contrary to the data in the literature, neither the loss of spontaneity during sexual relations, nor the complementary investigations necessary for artificial insemination had any influence on sexuality. $\mathbf{8 9 . 8 \%}$ of our patients are ready to change their sexual practices in order to be more fertile without causing any sexual or psychological problems. However, only $34.3 \%$ of patients reported a concern about reproductive efficacy during sexual relations. A more marked sexuality/reproduction dissociation was observed in oligoazoospermic males than in azoospermic males. $28.2 \%$ of patients reported that talking about their sexual and/or psychological problems with a psychiatrist was helpful.

Conc/usion: Many contradictions and underestimations were observed in the answers to the questions. We confirmed the existence of a psychologi- 
cal and sexual distress, but which was quantitatively less severe than that described in "gynaecological" populations. However, a real psychological and sexual distress does exist in our population, which is why we propose from the outset a psychosomatic and sexological treatment of infertility in order to prevent the onset of these sexual difficulties. Our questionnaire was based on clinical experience and helped our patients to identify their psychological and sexual difficulties related to infertility and its treatment. It would therefore be useful for the practitioner, andrologist or psychiatrist, to help the patient express the effects related to this "new medicalized sexuality".

Key words : sexuality, sterility, andrology, consultationliaison psychiatry

Manuscrit reçu : mai 2003 ; accepté août 2003. 